INLG 2019

\title{
The 12th International Conference on Natural Language Generation
}

\section{Proceedings of the Conference}

Oct 29 - Nov 1, 2019

Tokyo, Japan 
(C)2019 The Association for Computational Linguistics

Order copies of this and other ACL proceedings from:

Association for Computational Linguistics (ACL) 209 N. Eighth Street

Stroudsburg, PA 18360

USA

Tel: +1-570-476-8006

Fax: +1-570-476-0860

acleaclweb.org

ISBN 978-1-950737-94-9 


\section{Preface}

We are pleased to present the Proceedings of the 12th International Natural Language Generation Conference (INLG 2019), the first to be organised in Asia. The INLG conference is the main international forum for the discussion of all aspects of Natural Language Generation (NLG), including data-totext, concept-to-text, text-to-text and vision-to-text approaches. This year, we decided to reach out to a number of neighbouring research communities (including Machine Translation, Dialogue Systems, and Psycholinguistics, among others) by inviting a broad range of workshops, and by inviting keynote speakers from neighbouring communities as well.

INLG 2019 was organised by the Artificial Intelligence Research Center of Japan (AIRC, AIST), the University of Aberdeen, and Utrecht University. The event took place under the auspices of the Special Interest Group on Natural Language Generation (SIGGEN) of the Association for Computational Linguistics (ACL) and the International Speech Communication Association (ISCA).

We were very pleased to hear keynote presentations from the following experts:

- Philipp Koehn (Johns Hopkins University, USA)

- Kazunori Komatami (Osaka University, Japan)

- Roger van Gompel (University of Dundee, UK)

- Anya Belz (University of Brighton, UK)

In addition to the main conference, a number of workshops were accepted following an open Call for Workshop Proposals:

- 1st Workshop on Interactive Natural Language Technology for Explainable Artificial Intelligence

- 1st Workshop on AI Werewolf and Dialog System

- 4th Workshop on Computational Creativity in Language Generation

- 1st Workshop on Discourse Structure in Neural NLG

The rising trend in the number of submitted papers that we have seen for Natural Language Generation in the last few years continued strongly this year, so the main conference was extended to take up two and a half days. For the main conference, we received a record number of 147 submissions, of which 36 were accepted as long papers, 30 as short papers, 4 as demo papers, and 3 as generation challenge papers. All accepted papers for the main conference are included in these proceedings.

We received financial support from the following sponsors:

- ARRIA NLG

- Megagon Labs

- DENSO IT Lab

- Yahoo Japan

- Fuji Xerox 
- The Association for Natural Language Processing

- Google

- Facebook

We would like to thank all our sponsors for their support, and we extend our gratitude to all speakers and reviewers for their excellent work.

Kees van Deemter

Chenhgua Lin

Hiroya Takamura

INLG 2019 Programme Chairs 


\section{Programme Chairs:}

Kees van Deemter (Utrecht University, The Netherlands)

Chenghua Lin (University of Sheffield, UK)

Hiroya Takamura (AIST/Tokyo Institute of Technology, Japan)

\section{Invited Speakers:}

Philipp Koehn (Johns Hopkins University, USA)

Kazunori Komatami (Osaka University, Japan)

Roger van Gompel (University of Dundee, UK)

Anya Belz (University of Brighton, UK)

\section{Workshop Chairs:}

Wenge Rong (Beihang University, China)

Sho Takase (Tokyo Institute of Technology, Japan)

\section{Tutorial and Invited Speaker Chair:}

Riza Batista (University of Manchester, UK)

\section{Publicity Chair:}

Sebastian Gehrmann (Harvard University, US)

\section{Publication Chair:}

Xiao Li (University of Aberdeen, UK)

\section{Local Organization Committee:}

Takenobu Tokunaga (Chair, Tokyo Institute of Technology, Japan) Ichiro Kobayashi (Ochanomizu University, Japan)

Hitoshi Nishikawa (Tokyo Institute of Technology, Japan)

Hiroya Takamura (AIST/Tokyo Institute of Technology, Japan)

Sho Takase (Tokyo Institute of Technology, Japan)

Hikaru Yokono (Fujitsu Laboratories, Japan)

\section{Area Chairs:}

Albert Gatt (University of Malta)

Guanyi Chen (Utrecht University)

David Schlangen (University of Potsdam)

David McDonald (Smart Information Flow Technologies (dba SIFT, LLC))

Dimitra Gkatzia (Edinburgh Napier University)

Emiel Krahmer (Tilburg University) 
Alberto Bugarín Diz (Univ. Santiago de Compostela)

Ehud Reiter (University of Aberdeen)

Kathy McKeown (Columbia University)

Keith VanderLinden (Calvin College)

Michael White (The Ohio State University)

Pablo Duboue (Textualization Software Ltd.)

François Portet (Laboratoire d'Informatique de Grenoble)

Saad Mahamood (trivago N.V)

Kristina Striegnitz (Union College)

Mariët Theune (University of Twente)

\section{Program Committee:}

Manex Agirrezabal (University of Copenhagen)

Malihe Alikhani (Rutgers University)

Jose Alonso (University of Santiago de Compostela)

Jun Araki (Bosch Research)

Anja Belz (University of Brighton)

Jennifer Biggs (Defence Science and Technology Group)

Nadjet Bouayad-Agha (NLP Consultant)

Jan Buys (University of Washington)

Deng Cai (The Chinese University of Hong Kong)

Thiago Castro Ferreira (Tilburg University)

PohLian Choong (Commonwealth of Australia)

Yagmur Gizem Cinar (Naver Labs Europe)

Elizabeth Clark (University of Washington)

Brian Davis (Dublin City University)

Nina Dethlefs (University of Hull)

Ondřej Dušek (Charles University)

Zhenxin Fu (Peking University)

Lorenzo Gatti (Human Media Interaction, University of Twente)

Kim Gerdes (Sorbonne Nouvelle)

Martijn Goudbeek (Tilburg University)

Ting Han (National Institute of Advanced Industrial Science and Technology)

Wenjuan Han (ShanghaiTech University)

Aki Harma (Philips Research)

Sadid A. Hasan (Philips Research North America)

Hiroaki Hayashi (Carnegie Mellon University)

David M. Howcroft (Heriot-Watt University)

Wenpeng Hu (School of Mathematical Sciences, Peking University)

Amy Isard (University of Hamburg)

Feng Ji (Alibaba Group)

Aditya Joshi (CSIRO)

Natthawut Kertkeidkachorn (National Institute of Advanced Industrial Science and Technology)

Cyril Labbe (UGA)

Gerasimos Lampouras (University of Cambridge)

Hoa T. LE (Loria)

Chu-Cheng Lin (Johns Hopkins University)

Tianyu Liu (Peking University)

Elena Lloret (University of Alicante)

Pablo Loyola (IBM Research) 
Edison Marrese-Taylor (The University of Tokyo)

Prashant Mathur (Amazon)

Kathy McCoy (University of Delaware)

Antonio Valerio Miceli Barone (The University of Edinburgh)

Simon Mille (Pompeu Fabra University)

Diego Moussallem (Paderborn Univerisity)

Ryo Nagata (Konan University)

Jianmo Ni (University of California San Diego)

Xing Niu (University of Maryland)

Avinesh P.V.S (UKP Lab, Technische Universität Darmstadt)

Suraj Pandey (The Open University)

Cecile Paris (CSIRO)

Steffen Pauws (Philips Research)

Baolin Peng (The Chinese University of Hong Kong)

Minlong Peng (Fudan University)

Laura Perez-Beltrachini (School of Informatics, University of Edinburgh)

Paul Piwek (The Open University)

Shrimai Prabhumoye (Carnegie Mellon University)

Raheel Qader (Université Grenoble Alpes)

Alejandro Ramos Soto (CiTIUS, Universidade de Santiago de Compostela)

Wenge Rong (Beihang University)

Daniel Sanchez (University of Granada)

Mingyue Shang (Peking University)

Dinghan Shen (Duke University)

Lei Shu (Department of Computer Science, University of Illinois at Chicago)

Advaith Siddharthan (The Open University)

Linfeng Song (University of Rochester)

Balaji Vasan Srinivasan (Adobe Research)

Somayajulu Sripada (Arria NLG Plc and University of Aberdeen)

Shahbaz Syed (Leipzig University)

Hiroya Takamura (Tokyo Institute of Technology)

Hao Tan (The University of North Carolina at Chapel Hill)

Craig Thomson (University of Aberdeen)

Son Tran (University of Tasmania)

Lifu Tu (Toyota Technological Institute at Chicago)

Chris van der Lee (Tilburg University)

Emiel van Miltenburg (Tilburg University)

$\mathrm{Tu} \mathrm{Vu}$ (University of Massachusetts Amherst)

Michael Völske (Bauhaus-Universität Weimar)

Stephen Wan (CSIRO)

Qingyun Wang (University of Illinois at Urbana-Champaign)

Di Wang (School of Computer Science, Carnegie Mellon University)

Wenlin Wang (Duke University)

Leo Wanner (ICREA and Pompeu Fabra University)

Sander Wubben (Flow.ai / Tilburg University)

Qiongkai Xu (The Australian National University and Data61)

Chang Xu (Data61, CSIRO, Australia)

Pengcheng Yang (Peking University)

Jin-ge Yao (Peking University)

Sina Zarrieß (University of Jena)

Zhirui Zhang (University of Science and Technology of China) 
Zaixiang Zheng (National Key Laboratory for Novel Software Technology, Nanjing University) Yinhe Zheng (Tsinghua University)

Qingyu Zhou (Harbin Institute of Technology)

Qile Zhu (University of florida) 


\section{Table of Contents}

Talking about what is not there: Generating indefinite referring expressions in Minecraft

Arne Köhn and Alexander Koller........................................ 1

Generating Quantified Referring Expressions with Perceptual Cost Pruning

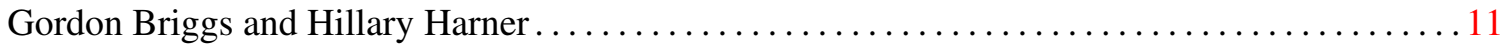

A case study on context-bound referring expression generation

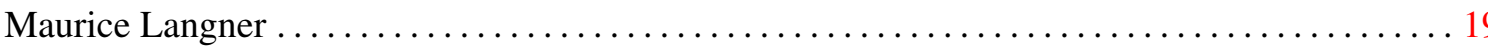

Rethinking Text Attribute Transfer: A Lexical Analysis

Yao Fu, Hao Zhou, Jiaze Chen and Lei Li ................................. 24

Choosing between Long and Short Word Forms in Mandarin

Lin Li, Kees van Deemter, Denis Paperno and Jingyu Fan......................... 34

Diamonds in the Rough: Generating Fluent Sentences from Early-Stage Drafts for Academic Writing Assistance

Takumi Ito, Tatsuki Kuribayashi, Hayato Kobayashi, Ana Brassard, Masato Hagiwara, Jun Suzuki

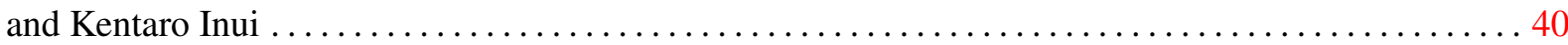

Computational Argumentation Synthesis as a Language Modeling Task

Roxanne El Baff, Henning Wachsmuth, Khalid Al Khatib, Manfred Stede and Benno Stein ... 54

Towards Coherent and Engaging Spoken Dialog Response Generation Using Automatic Conversation Evaluators

Sanghyun Yi, Rahul Goel, Chandra Khatri, Alessandra Cervone, Tagyoung Chung, Behnam Hedayatnia, Anu Venkatesh, Raefer Gabriel and Dilek Hakkani-Tur. . . . . . . . . . . . . . . . . . . 65

Importance of Search and Evaluation Strategies in Neural Dialogue Modeling

Ilia Kulikov, Alexander Miller, Kyunghyun Cho and Jason Weston...................... 76

Towards Best Experiment Design for Evaluating Dialogue System Output

Sashank Santhanam and Samira Shaikh ................................... 88

A Tree-to-Sequence Model for Neural NLG in Task-Oriented Dialog

Jinfeng Rao, Kartikeya Upasani, Anusha Balakrishnan, Michael White, Anuj Kumar and Rajen

Subba...................................................... 95

Multiple News Headlines Generation using Page Metadata

Kango Iwama and Yoshinobu Kano ..................................... 101

Neural Question Generation using Interrogative Phrases

Yuichi Sasazawa, Sho Takase and Naoaki Okazaki ............................. 106

Generating Text from Anonymised Structures

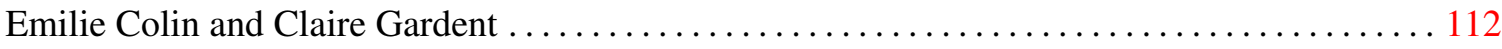

MinWikiSplit: A Sentence Splitting Corpus with Minimal Propositions

Christina Niklaus, André Freitas and Siegfried Handschuh ......................... 118

QTUNA: A Corpus for Understanding How Speakers Use Quantification

Guanyi Chen, Kees van Deemter, Silvia Pagliaro, Louk Smalbil and Chenghua Lin.......... 124 
KPTimes: A Large-Scale Dataset for Keyphrase Generation on News Documents

Ygor Gallina, Florian Boudin and Beatrice Daille............................. 130

Sketch Me if You Can: Towards Generating Detailed Descriptions of Object Shape by Grounding in Images and Drawings

Ting Han and Sina Zarrieß

An Encoder with non-Sequential Dependency for Neural Data-to-Text Generation

Feng Nie, Jinpeng Wang, Rong Pan and Chin-Yew Lin ...

On Leveraging the Visual Modality for Neural Machine Translation

Vikas Raunak, Sang Keun Choe, Quanyang Lu, Yi Xu and Florian Metze

Tell Me More: A Dataset of Visual Scene Description Sequences

Nikolai Ilinykh, Sina Zarrieß and David Schlangen ...

A Closer Look at Recent Results of Verb Selection for Data-to-Text NLG

Guanyi Chen and Jin-Ge Yao

ViGGO: A Video Game Corpus for Data-To-Text Generation in Open-Domain Conversation

Juraj Juraska, Kevin Bowden and Marilyn Walker............................... 164

BERT for Question Generation

Ying-Hong Chan and Yao-Chung Fan.

Visually grounded generation of entailments from premises

Somayeh Jafaritazehjani, Albert Gatt and Marc Tanti .

Detecting Machine-Translated Text using Back Translation

Hoang-Quoc Nguyen-Son, Thao Tran Phuong, Seira Hidano and Shinsaku Kiyomoto

Neural Conversation Model Controllable by Given Dialogue Act Based on Adversarial Learning and Label-aware Objective

Seiya Kawano, Koichiro Yoshino and Satoshi Nakamura 198

Low Level Linguistic Controls for Style Transfer and Content Preservation

Katy Gero, Chris Kedzie, Jonathan Reeve and Lydia Chilton ........................ 208

Selecting Artificially-Generated Sentences for Fine-Tuning Neural Machine Translation

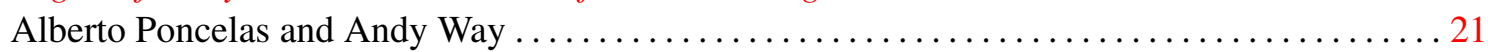

Efficiency Metrics for Data-Driven Models: A Text Summarization Case Study

Erion Çano and Ondřej Bojar ...

An NLG System for Constituent Correspondence: Personality, Affect, and Alignment

William Kolkey, Jian Dong and Greg Bybee...

Margin Call: an Accessible Web-based Text Viewer with Generated Paragraph Summaries in the Margin

Nabah Rizvi, Sebastian Gehrmann and Franck Dernoncourt........................ 244

Privacy-Aware Text Rewriting

Qiongkai Xu, Lizhen Qu, Chenchen Xu and Ran Cui ...

Personalized Substitution Ranking for Lexical Simplification

John Lee and Chak Yan Yeung. ... 
Revisiting the Binary Linearization Technique for Surface Realization

Yevgeniy Puzikov, Claire Gardent, Ido Dagan and Iryna Gurevych.................... 268

Head-First Linearization with Tree-Structured Representation

Xiang Yu, Agnieszka Falenska, Ngoc Thang Vu and Jonas Kuhn ..................... 279

Let's FACE it. Finnish Poetry Generation with Aesthetics and Framing

Mika Hämäläinen and Khalid Alnajjar ..................................... 290

Generation of Hip-Hop Lyrics with Hierarchical Modeling and Conditional Templates

Enrique Manjavacas, Mike Kestemont and Folgert Karsdorp ....................... 301

Revisiting Challenges in Data-to-Text Generation with Fact Grounding

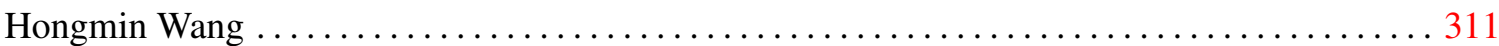

Controlling Contents in Data-to-Document Generation with Human-Designed Topic Labels

Kasumi Aoki, Akira Miyazawa, Tatsuya Ishigaki, Tatsuya Aoki, Hiroshi Noji, Keiichi Goshima, Ichiro Kobayashi, Hiroya Takamura and Yusuke Miyao ........................... 323

A Large-Scale Multi-Length Headline Corpus for Analyzing Length-Constrained Headline Generation Model Evaluation

Yuta Hitomi, Yuya Taguchi, Hideaki Tamori, Ko Kikuta, Jiro Nishitoba, Naoaki Okazaki, Kentaro

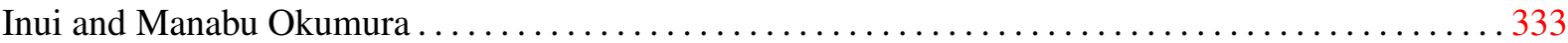

Agreement is overrated: A plea for correlation to assess human evaluation reliability

Jacopo Amidei, Paul Piwek and Alistair Willis................................... 344

Best practices for the human evaluation of automatically generated text

Chris van der Lee, Albert Gatt, Emiel van Miltenburg, Sander Wubben and Emiel Krahmer ... 355

Automatic Quality Estimation for Natural Language Generation: Ranting (Jointly Rating and Ranking)

Ondřej Dušek, Karin Sevegnani, Ioannis Konstas and Verena Rieser ..................... 369

Improving Quality and Efficiency in Plan-based Neural Data-to-text Generation

Amit Moryossef, Yoav Goldberg and Ido Dagan ............................ 377

Toward a Better Story End: Collecting Human Evaluation with Reasons

Yusuke Mori, Hiroaki Yamane, Yusuke Mukuta and Tatsuya Harada ................... 383

Hotel Scribe: Generating High Variation Hotel Descriptions

Saad Mahamood and Maciej Zembrzuski

The use of rating and Likert scales in Natural Language Generation human evaluation tasks: A review and some recommendations

Jacopo Amidei, Paul Piwek and Alistair Willis.

On task effects in NLG corpus elicitation: a replication study using mixed effects modeling

Emiel van Miltenburg, Merel van de Kerkhof, Ruud Koolen, Martijn Goudbeek and Emiel Krahmer 403

Procedural Text Generation from a Photo Sequence

Taichi Nishimura, Atsushi Hashimoto and Shinsuke Mori. . .

SimpleNLG-DE: Adapting SimpleNLG 4 to German

Daniel Braun, Kira Klimt, Daniela Schneider and Florian Matthes 
Semantic Noise Matters for Neural Natural Language Generation

Ondřej Dušek, David M. Howcroft and Verena Rieser .......................... 421

Can Neural Image Captioning be Controlled via Forced Attention?

Philipp Sadler, Tatjana Scheffler and David Schlangen.......................... 427

Towards a Metric for Automated Conversational Dialogue System Evaluation and Improvement

Jan Milan Deriu and Mark Cieliebak ................................... 432

Generating Paraphrases with Lean Vocabulary

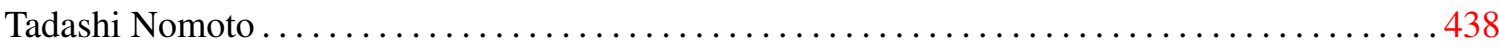

A Personalized Data-to-Text Support Tool for Cancer Patients

Saar Hommes, Chris van der Lee, Felix Clouth, Jeroen Vermunt, Xander Verbeek and Emiel Krahmer .............................................................. 443

Natural Language Generation at Scale: A Case Study for Open Domain Question Answering

Alessandra Cervone, Chandra Khatri, Rahul Goel, Behnam Hedayatnia, Anu Venkatesh, Dilek Hakkani-Tur and Raefer Gabriel ......................................... 453

Using NLG for speech synthesis of mathematical sentences

Alessandro Mazzei, Michele Monticone and Cristian Bernareggi .................... 463

Teaching FORGe to Verbalize DBpedia Properties in Spanish

Simon Mille, Stamatia Dasiopoulou, Beatriz Fisas and Leo Wanner

Generating justifications for norm-related agent decisions

Daniel Kasenberg, Antonio Roque, Ravenna Thielstrom, Meia Chita-Tegmark and Matthias Scheutz 484

Towards Generating Math Word Problems from Equations and Topics

Qingyu Zhou and Danqing Huang....

DisSim: A Discourse-Aware Syntactic Text Simplification Framework for English and German

Christina Niklaus, Matthias Cetto, André Freitas and Siegfried Handschuh ............... 504

Real World Voice Assistant System for Cooking

Takahiko Ito, Shintaro Inuzuka, Yoshiaki Yamada and Jun Harashima.................. 508

VAE-PGN based Abstractive Model in Multi-stage Architecture for Text Summarization

Hyungtak Choi, Lohith Ravuru, Tomasz Dryjanski, Sunghan Rye, Donghyun Lee, Hojung Lee and

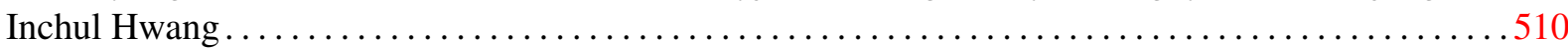

Generating Abstractive Summaries with Finetuned Language Models

Sebastian Gehrmann, Zachary Ziegler and Alexander Rush ........................ 516

Towards Summarization for Social Media - Results of the TL;DR Challenge

Shahbaz Syed, Michael Völske, Nedim Lipka, Benno Stein, Hinrich Schütze and Martin Potthast 523

Generating Quantified Descriptions of Abstract Visual Scenes

Guanyi Chen, Kees van Deemter and Chenghua Lin............................. 529

What goes into a word: generating image descriptions with top-down spatial knowledge

Mehdi Ghanimifard and Simon Dobnik .................................... 540 
Semi-Supervised Neural Text Generation by Joint Learning of Natural Language Generation and Natural Language Understanding Models

Raheel Qader, François Portet and Cyril Labbé

Neural Generation for Czech: Data and Baselines

Ondřej Dušek and Filip Jurčíček

Modeling Confidence in Sequence-to-Sequence Models

Jan Niehues and Ngoc-Quan Pham . . 575

A Good Sample is Hard to Find: Noise Injection Sampling and Self-Training for Neural Language Generation Models

Chris Kedzie and Kathleen McKeown 584

A Stable Variational Autoencoder for Text Modelling

Ruizhe Li, Xiao Li, Chenghua Lin, Matthew Collinson and Rui Mao. . 594 



\section{Conference Program}

29 Oct 2019

10:30-13:30 Workshop 1: Interactive Natural Language Technology for Explainable Artificial Intelligence (NL4XAI2019)

10:30-13:10 Workshop 2: AI Werewolf and Dialog System (AIWolfDial2019)

14:30-14:45 Opening of INLG 2019

14:45-15:35 Tutorial 2: Deepfake News Generation: Methods, Detection and Wider Implications Anya Belz

15:50-16:50 Oral Session 1: Referring Expression Generation

Talking about what is not there: Generating indefinite referring expressions in Minecraft

Arne Köhn and Alexander Koller

Generating Quantified Referring Expressions with Perceptual Cost Pruning

Gordon Briggs and Hillary Harner

A case study on context-bound referring expression generation

Maurice Langner 


\section{Oct 2019 (continued)}

\section{7:05-18:05 Oral Session 2: Generation Techniques 1}

Rethinking Text Attribute Transfer: A Lexical Analysis

Yao Fu, Hao Zhou, Jiaze Chen and Lei Li

Choosing between Long and Short Word Forms in Mandarin

Lin Li, Kees van Deemter, Denis Paperno and Jingyu Fan

Diamonds in the Rough: Generating Fluent Sentences from Early-Stage Drafts for Academic Writing Assistance

Takumi Ito, Tatsuki Kuribayashi, Hayato Kobayashi, Ana Brassard, Masato Hagiwara, Jun Suzuki and Kentaro Inui

\section{Oct 2019}

10:00-10:50 Tutorial 2: User-Aware Dialogue Systems with Adaptive Responses Kazunori Komatani

\section{1:05-12:25 Oral Session 3: Dialogue and Argumentation}

Computational Argumentation Synthesis as a Language Modeling Task

Roxanne El Baff, Henning Wachsmuth, Khalid Al Khatib, Manfred Stede and Benno Stein

Towards Coherent and Engaging Spoken Dialog Response Generation Using Automatic Conversation Evaluators

Sanghyun Yi, Rahul Goel, Chandra Khatri, Alessandra Cervone, Tagyoung Chung, Behnam Hedayatnia, Anu Venkatesh, Raefer Gabriel and Dilek Hakkani-Tur

Importance of Search and Evaluation Strategies in Neural Dialogue Modeling Ilia Kulikov, Alexander Miller, Kyunghyun Cho and Jason Weston

Towards Best Experiment Design for Evaluating Dialogue System Output Sashank Santhanam and Samira Shaikh 


\section{Oct 2019 (continued)}

\section{2:25-13:50 Poster Session 1}

A Tree-to-Sequence Model for Neural NLG in Task-Oriented Dialog

Jinfeng Rao, Kartikeya Upasani, Anusha Balakrishnan, Michael White, Anuj Kumar and Rajen Subba

Multiple News Headlines Generation using Page Metadata

Kango Iwama and Yoshinobu Kano

Neural Question Generation using Interrogative Phrases

Yuichi Sasazawa, Sho Takase and Naoaki Okazaki

Generating Text from Anonymised Structures

Emilie Colin and Claire Gardent

MinWikiSplit: A Sentence Splitting Corpus with Minimal Propositions

Christina Niklaus, André Freitas and Siegfried Handschuh

QTUNA: A Corpus for Understanding How Speakers Use Quantification

Guanyi Chen, Kees van Deemter, Silvia Pagliaro, Louk Smalbil and Chenghua Lin

KPTimes: A Large-Scale Dataset for Keyphrase Generation on News Documents Ygor Gallina, Florian Boudin and Beatrice Daille

Sketch Me if You Can: Towards Generating Detailed Descriptions of Object Shape by Grounding in Images and Drawings

Ting Han and Sina Zarrieß

An Encoder with non-Sequential Dependency for Neural Data-to-Text Generation Feng Nie, Jinpeng Wang, Rong Pan and Chin-Yew Lin

On Leveraging the Visual Modality for Neural Machine Translation

Vikas Raunak, Sang Keun Choe, Quanyang Lu, Yi Xu and Florian Metze

Tell Me More: A Dataset of Visual Scene Description Sequences

Nikolai Ilinykh, Sina Zarrieß and David Schlangen 


\section{Oct 2019 (continued)}

A Closer Look at Recent Results of Verb Selection for Data-to-Text NLG

Guanyi Chen and Jin-Ge Yao

ViGGO: A Video Game Corpus for Data-To-Text Generation in Open-Domain Conversation

Juraj Juraska, Kevin Bowden and Marilyn Walker

BERT for Question Generation

Ying-Hong Chan and Yao-Chung Fan

Visually grounded generation of entailments from premises

Somayeh Jafaritazehjani, Albert Gatt and Marc Tanti

Detecting Machine-Translated Text using Back Translation

Hoang-Quoc Nguyen-Son, Thao Tran Phuong, Seira Hidano and Shinsaku Kiyomoto

Neural Conversation Model Controllable by Given Dialogue Act Based on Adversarial Learning and Label-aware Objective

Seiya Kawano, Koichiro Yoshino and Satoshi Nakamura

Low Level Linguistic Controls for Style Transfer and Content Preservation

Katy Gero, Chris Kedzie, Jonathan Reeve and Lydia Chilton

Selecting Artificially-Generated Sentences for Fine-Tuning Neural Machine Translation

Alberto Poncelas and Andy Way

Efficiency Metrics for Data-Driven Models: A Text Summarization Case Study

Erion Çano and Ondřej Bojar

An NLG System for Constituent Correspondence: Personality, Affect, and Alignment William Kolkey, Jian Dong and Greg Bybee

Margin Call: an Accessible Web-based Text Viewer with Generated Paragraph Summaries in the Margin

Nabah Rizvi, Sebastian Gehrmann and Franck Dernoncourt 


\section{Oct 2019 (continued)}

\section{3:50-15:20 GenChal}

\section{5:40-17:00 Oral Session 4: Generation Techniques 2}

Privacy-Aware Text Rewriting

Qiongkai Xu, Lizhen Qu, Chenchen Xu and Ran Cui

Personalized Substitution Ranking for Lexical Simplification

John Lee and Chak Yan Yeung

Revisiting the Binary Linearization Technique for Surface Realization

Yevgeniy Puzikov, Claire Gardent, Ido Dagan and Iryna Gurevych

Head-First Linearization with Tree-Structured Representation

Xiang Yu, Agnieszka Falenska, Ngoc Thang Vu and Jonas Kuhn

\section{7:10-18:30 Oral Session 5: Creative and Controllable Text Generation}

Let's FACE it. Finnish Poetry Generation with Aesthetics and Framing

Mika Hämäläinen and Khalid Alnajjar

Generation of Hip-Hop Lyrics with Hierarchical Modeling and Conditional Templates

Enrique Manjavacas, Mike Kestemont and Folgert Karsdorp

Revisiting Challenges in Data-to-Text Generation with Fact Grounding

Hongmin Wang

Controlling Contents in Data-to-Document Generation with Human-Designed Topic Labels

Kasumi Aoki, Akira Miyazawa, Tatsuya Ishigaki, Tatsuya Aoki, Hiroshi Noji, Keiichi Goshima, Ichiro Kobayashi, Hiroya Takamura and Yusuke Miyao 


\section{Oct 2019}

10:00-10:50 Tutorial 3: New Challenges for Neural Sequence Generation Models - Insights from Machine Translation

Philipp Koehn

\section{1:05-12:25 Oral Session 6: Evaluation and Language Resources}

A Large-Scale Multi-Length Headline Corpus for Analyzing Length-Constrained Headline Generation Model Evaluation

Yuta Hitomi, Yuya Taguchi, Hideaki Tamori, Ko Kikuta, Jiro Nishitoba, Naoaki

Okazaki, Kentaro Inui and Manabu Okumura

Agreement is overrated: A plea for correlation to assess human evaluation reliability

Jacopo Amidei, Paul Piwek and Alistair Willis

Best practices for the human evaluation of automatically generated text

Chris van der Lee, Albert Gatt, Emiel van Miltenburg, Sander Wubben and Emiel Krahmer

Automatic Quality Estimation for Natural Language Generation: Ranting (Jointly Rating and Ranking)

Ondřej Dušek, Karin Sevegnani, Ioannis Konstas and Verena Rieser

\section{2:25-13:50 Poster Session 2}

Improving Quality and Efficiency in Plan-based Neural Data-to-text Generation Amit Moryossef, Yoav Goldberg and Ido Dagan

Toward a Better Story End: Collecting Human Evaluation with Reasons

Yusuke Mori, Hiroaki Yamane, Yusuke Mukuta and Tatsuya Harada

Hotel Scribe: Generating High Variation Hotel Descriptions

Saad Mahamood and Maciej Zembrzuski

The use of rating and Likert scales in Natural Language Generation human evaluation tasks: A review and some recommendations

Jacopo Amidei, Paul Piwek and Alistair Willis

On task effects in NLG corpus elicitation: a replication study using mixed effects modeling

Emiel van Miltenburg, Merel van de Kerkhof, Ruud Koolen, Martijn Goudbeek and Emiel Krahmer 


\section{Oct 2019 (continued)}

Procedural Text Generation from a Photo Sequence

Taichi Nishimura, Atsushi Hashimoto and Shinsuke Mori

SimpleNLG-DE: Adapting SimpleNLG 4 to German

Daniel Braun, Kira Klimt, Daniela Schneider and Florian Matthes

Semantic Noise Matters for Neural Natural Language Generation

Ondřej Dušek, David M. Howcroft and Verena Rieser

Can Neural Image Captioning be Controlled via Forced Attention?

Philipp Sadler, Tatjana Scheffler and David Schlangen

Towards a Metric for Automated Conversational Dialogue System Evaluation and Improvement

Jan Milan Deriu and Mark Cieliebak

Generating Paraphrases with Lean Vocabulary

Tadashi Nomoto

A Personalized Data-to-Text Support Tool for Cancer Patients

Saar Hommes, Chris van der Lee, Felix Clouth, Jeroen Vermunt, Xander Verbeek and Emiel Krahmer

Natural Language Generation at Scale: A Case Study for Open Domain Question Answering

Alessandra Cervone, Chandra Khatri, Rahul Goel, Behnam Hedayatnia, Anu Venkatesh, Dilek Hakkani-Tur and Raefer Gabriel

Using NLG for speech synthesis of mathematical sentences

Alessandro Mazzei, Michele Monticone and Cristian Bernareggi

Teaching FORGe to Verbalize DBpedia Properties in Spanish

Simon Mille, Stamatia Dasiopoulou, Beatriz Fisas and Leo Wanner

Generating justifications for norm-related agent decisions

Daniel Kasenberg, Antonio Roque, Ravenna Thielstrom, Meia Chita-Tegmark and Matthias Scheutz

Towards Generating Math Word Problems from Equations and Topics

Qingyu Zhou and Danqing Huang 


\section{Oct 2019 (continued)}

DisSim: A Discourse-Aware Syntactic Text Simplification Framework for English and German

Christina Niklaus, Matthias Cetto, André Freitas and Siegfried Handschuh

Real World Voice Assistant System for Cooking

Takahiko Ito, Shintaro Inuzuka, Yoshiaki Yamada and Jun Harashima

VAE-PGN based Abstractive Model in Multi-stage Architecture for Text Summarization

Hyungtak Choi, Lohith Ravuru, Tomasz Dryjanski, Sunghan Rye, Donghyun Lee, Hojung Lee and Inchul Hwang

Generating Abstractive Summaries with Finetuned Language Models Sebastian Gehrmann, Zachary Ziegler and Alexander Rush

Towards Summarization for Social Media - Results of the TL;DR Challenge Shahbaz Syed, Michael Völske, Nedim Lipka, Benno Stein, Hinrich Schütze and Martin Potthast

\section{3:50-14:30 Oral Session 7: Describnig Visual Scenes}

Generating Quantified Descriptions of Abstract Visual Scenes

Guanyi Chen, Kees van Deemter and Chenghua Lin

What goes into a word: generating image descriptions with top-down spatial knowledge

Mehdi Ghanimifard and Simon Dobnik

14:30-15:20 Tutorial 4: Developing Psychologically Plausible NLG Models of Human Reference Production

Roger van Gompel 


\section{Oct 2019 (continued)}

\section{5:35-16:55 Oral Session 8: Neural Language Generation}

Semi-Supervised Neural Text Generation by Joint Learning of Natural Language Generation and Natural Language Understanding Models

Raheel Qader, François Portet and Cyril Labbé

Neural Generation for Czech: Data and Baselines

Ondřej Dušek and Filip Jurčíček

Modeling Confidence in Sequence-to-Sequence Models

Jan Niehues and Ngoc-Quan Pham

A Good Sample is Hard to Find: Noise Injection Sampling and Self-Training for Neural Language Generation Models

Chris Kedzie and Kathleen McKeown

A Stable Variational Autoencoder for Text Modelling

Ruizhe Li, Xiao Li, Chenghua Lin, Matthew Collinson and Rui Mao

1 Nov 2019

10:00-13:00 Workshop 3: Discourse Structure in Neural NLG

10:00-13:00 Workshop 4: Computational Creativity in Language Generation 
1 Nov 2019 (continued)

14:30-15:45 Workshop 5: Discourse Structure in Neural NLG

14:30-15:45 workshop 6: Computational Creativity in Language Generation 